\title{
Correction to: Interparental Interactions and Adolescent Mood: A Daily Diary Approach
}

\author{
Christine E. Merrilees ${ }^{1} \cdot$ Meghan P. McCormick ${ }^{2} \cdot$ JoAnn Hsueh $^{2} \cdot$ Patricia Chou $^{2} \cdot$ E. Mark Cummings ${ }^{3}$
}

Published online: 6 March 2018

(c) Springer Science+Business Media, LLC, part of Springer Nature 2018

Correction to: Journal of Child and Family Study (2018) https://doi.org/10.1007/s10826-017-0983-2

The original version of this article unfortunately contained mistakes. The affiliation of the first author was included as one of the authors and the affiliations were incorrect. They are now corrected with this erratum. The original article has been corrected.
${ }^{1}$ Psychology Department, SUNY Geneseo, Geneseo, NY, USA

${ }^{2}$ Family and Children Policy Area, MDRC, New York, NY, USA

${ }^{3}$ Department of Psychology, University of Notre Dame, Notre Dame, IN, USA

The original article can be found online at https://doi.org/10.1007/ s10826-017-0983-2.

Christine E. Merrilees merrilees@geneseo.edu

1 Psychology Department, SUNY Geneseo, Geneseo, NY, USA

2 Family and Children Policy Area, MDRC, New York, NY, USA

3 Department of Psychology, University of Notre Dame,

Notre Dame, IN, USA 